\title{
Análise fractal da fragmentação por impacto de porcelanas
}

\section{(Fractal analysis of fragmentation by impact of porcelains)}

\author{
C. B. Mvumbi, A. L. Chinelatto, A. S. A. Chinelatto, L. Máximo Alves \\ Programa de Mestrado em Engenharia e Ciência de Materiais, Universidade Estadual de Ponta Grossa, \\ Av. Carlos Cavalcanti 4748, Ponta Grossa, PR 84030-900 \\ charlesbetuel1@hotmail.com,adilson@uepg.br,adriana@uepg.br,lucasmaximoalves@gmail.com
}

\begin{abstract}
Resumo
As porcelanas são cerâmicas produzidas a partir de matérias-primas naturais (sílica, feldspato e caulim). Em algumas aplicações são adicionadas outras matérias-primas para o aumento da resistência mecânica e dielétrica. Elas são usadas na fabricação de variados produtos, sendo, portanto, muito estudadas e desenvolvidas há muito tempo. Neste trabalho, porcelanas com diferentes composições foram caracterizadas e submetidas à fragmentação por ruptura mecânica em um ensaio de impacto balístico. A ruptura mecânica nos materiais em geral produz uma dissipação de energia formando trincas e superfícies de fratura que tem uma geometria irregular. O principal objetivo deste trabalho foi a caracterização da microestrutura e a caracterização fractal da superfície de fratura de porcelanas. Para comparação, os ensaios de impacto também foram feitos em vidro e acrílico comercial. A avaliação da superfície de fratura e da dimensão fractal de ruptura foi feita pela análise da fração de fragmentos em função da probabilidade global de fratura. Os corpos de prova foram preparados na forma de discos cilíndricos, com espessura e diâmetros fixos. O ensaio de impacto mecânico foi feito sobre o centro dos discos, para os diferentes materiais, mantendo-se as mesmas condições de ensaio. Os resultados do tamanho e massa dos fragmentos foram obtidos em função da energia de impacto e do tipo de material. Desta forma, foi possível obter uma correlação entre as condições de ensaio e a composição do material com o padrão de ruptura fractal. Verificou-se que a porcelana nas composições usadas possuiu uma tenacidade ao impacto que se situou entre aquela do vidro e do acrílico. Finalmente foi feita uma correlação entre as propriedades de resistência mecânica com os padrões de trinca formados. Percebeu-se que o número, a forma das trincas e as ramificações formaram um padrão que foi relacionado com a energia de impacto e o tipo de material.
\end{abstract}

Palavras-chave: porcelana, ruptura mecânica, dimensão fractal, impacto central.

\begin{abstract}
The ceramic porcelains are produced from natural raw materials (silica, feldspar and kaolin). In some applications, other materials are added to increase the mechanical and dielectric strength. They are used in the manufacture of several products. Therefore, they are very studied and have been developed for long time. In the work herein porcelains with different compositions were characterized and submitted to fragmentation by mechanical rupture in a ballistic impact test. Generally, the mechanical disruption in the materials produces a power dissipation forming cracks and fracture surfaces having an irregular geometry. The main objective of the work herein was to characterize the microstructure and the fractal fracture surface in porcelains. For comparison, the impact tests were also performed on commercial glass and acrylic. The evaluation of the fracture surface and the fractal dimension of rupture were performed by analyzing the fraction of the fragments as a function of the probability of fracture. The samples were prepared in the form of cylindrical disks with fixed thickness and diameter values. Mechanical impact test was applied on the center of the disks for different materials, maintaining the same test conditions. The results of the size and mass of the fragments were obtained as a function of the impact energy and kind of material. Thus, it was possible to obtain a correlation between the test conditions and the composition of the material and the fractal cracking pattern. It has been found that the compositions used in porcelain presented a fracture toughness which was between the one of the glass and of the acrylic. A correlation between strength properties and the patterns of formed crack was verified. It was noticed that the number, the shape of cracks and the branches formed a pattern that was associated with the impact energy and material type.
\end{abstract}

Keywords: porcelain, mechanical rupture, fractal dimension, central impact.

\section{INTRODUÇÃO}

Basicamente, as porcelanas são produzidas a partir de matérias-primas naturais, que são a argila plástica, o feldspato e o quartzo e/ou alumina e, em alguns casos, são adicionados outros compostos para alterar algumas propriedades específicas. As porcelanas são cerâmicas policristali- nas com uma textura fina, que podem conter de 50 a $90 \%$ de volume contínuo na sua fase vítrea, sendo este responsável pela unificação de suas partículas cristalinas durante a queima. Esta unificação ajuda na translucidez do material e no grande aumento da sua resistência mecânica e rigidez dielétrica nas chamadas porcelanas elétricas [1]. A substituição parcial ou total do quartzo por alumina em alguns 
tipos de massas de porcelana tem sido utilizada para promover aumento da resistência mecânica [2, 3]. As porcelanas formadas por alumina, feldspato e caulim são chamadas de porcelanas aluminosas e são utilizadas na fabricação de isoladores elétricos de alta tensão, por possuírem propriedades dielétricas adequadas e propriedades mecânicas superiores às das porcelanas que utilizam o quartzo $[4,5]$. Em porcelanas contendo alumina, a resistência mecânica é dependente do tamanho e forma das partículas de alumina dispersas na microestrutura [6]. Além disso, o módulo elástico da alumina é maior do que o do quartzo, o que também induz um aumento de resistência mecânica [7].

A ruptura mecânica por impacto de matérias frágeis, como a porcelana, produz um padrão de dissipação de energia formado por trincas que seguem uma geometria irregular. A geometria irregular das trincas pode ser bem caracterizada pela geometria fractal. A caracterização fractal, por sua vez, permite classificar as estruturas de dissipação por meio da dimensão fractal de ruptura. Portanto, uma ruptura mecânica pode ser avaliada por meio da sua dimensão fractal, possibilitando caracterizar as diferentes condições de ensaio dadas pela composição do material, tensão de ruptura e dimensões da amostra, as quais vão se refletir no padrão de trincas [8]. A probabilidade local de fratura é definida como a fração da massa dos fragmentos, $p=m / m_{0}$, onde $m$ é a massa de cada fragmento e $m_{0}$ é a massa total do corpo de prova. A probabilidade global de fratura é definida como sendo a porcentagem de fragmentos acima de um determinado tamanho, obtida em um ensaio de impacto de um disco de um material com diâmetro e espessura determinados, submetido a uma dada quantidade de energia de impacto. Portanto, essa probabilidade global de fratura, $\mathrm{P}_{\mathrm{f}}$, depende do material e da geometria do corpo de prova $[9,10]$, ou seja:

$$
\mathrm{P}_{\mathrm{f}}=\mathrm{f} \text { (material, geometria, energia) }
$$

A função densidade de probabilidade de fratura pode ser extraída experimentalmente a partir de medidas realizadas em discos de materiais frágeis, por exemplo. Desta forma, para um dado material com densidade uniforme, isto é $\rho=d m / d V=m_{0} / V$, pode-se escrever:

$$
\mathrm{P}_{\mathrm{f}}=\frac{1}{\mathrm{~V}} \int \mathrm{pdV}
$$

em que, $p$ é a probabilidade local de fratura, $d V$ é o diferencial do volume do disco, e $V=\pi r^{2} e$, onde $r$ é o raio do disco e $e$ é a sua espessura. O resultado do experimento fornece um histograma de frequência de massa em função do tamanho relativo dos fragmentos que corresponde à densidade de probabilidade local de fratura. $\mathrm{O}$ estudo experimental desses histogramas pode fornecer subsídio para o modelo multifractal da fratura, no qual as múltiplas dimensões fractais dos fragmentos variam dentro de um espectro de dimensões e as probabilidades locais e globais de fratura são consideradas.

Segundo $[9,10]$, a teoria de fragmentação sequencial é dada por duas escalas, fragmentação e refragmentação. Isto consiste na sequência que os modelos de escalas são usados na discrepância da análise da distribuição de massa a partir das experiências de fragmentação. De acordo com esta teoria, a fragmentação sequencial é dada por [10]:

$$
\mathrm{n}(\mathrm{m})=\mathrm{A} \int_{\mathrm{m}}^{\infty} \mathrm{n}\left(\mathrm{m}_{0}\right) \mathrm{f}\left(\mathrm{m}_{0} \rightarrow \mathrm{m}\right) d \mathrm{~m}_{0}
$$

em que se define $n(m)$ como o número de fragmentos após a ruptura por unidade de massa inicial $m_{0}$ até a massa final, sendo que $f\left(m_{0} \rightarrow m\right)$ é a função estatística da massa $m$ dos fragmentos, correspondendo a um único evento de fragmentação, que acontece por apenas um único impacto. Considerando-se que a função estatística é dada pela relação de escala entre a massa fragmentada e a massa inicial $m_{0}$, então:

$$
\mathrm{f}\left(\mathrm{m}_{0} \rightarrow \mathrm{m}\right)=\left(\frac{\mathrm{m}}{\mathrm{m}_{0}}\right)^{\gamma}
$$

sendo $\gamma$ o expoente fractal da fragmentação. Substituindo a constante $A$ da Equação $\mathrm{C}$, dada em termos da razão entre a massa inicial e a massa final do processo de fragmentação, obtém-se a fração de fragmentos, $F(>m)$, maiores do que uma quantidade de massa $m$, que também corresponde à probabilidade de sobrevivência a ruptura $P(s)$ :

$$
\mathrm{F}(>\mathrm{m}): \mathrm{P}(\mathrm{s})=\exp \left[-\frac{\left(\mathrm{m} / \mathrm{m}_{0}\right)^{\gamma+1}}{\gamma+1}\right]
$$

A partir da Equação E, que fornece a probabilidade de sobrevivência à ruptura para fragmentos maiores que um determinado tamanho relativo da massa $\mathrm{m} / \mathrm{m}_{0}$, pode-se obter a probabilidade de falha $P(f)$ para a fração de fragmentos menores ou iguais que um valor de massa, $F(\leq m)$, também em função da massa relativa dos fragmentos. A probabilidade de falha $P(f)$ é o resultado obtido diretamente pelo ensaio de impacto na forma de um histograma. Esse histograma é dado pela fração de fragmentos menores que um determinado tamanho ou massa, $F(\leq m)$ em função da massa relativa dos fragmentos $p_{i}=m_{i} / m_{0}$, onde $i$ é um índice genérico usado na indexação dos fragmentos em ordem crescente de massa. Comparado a distribuição de Weibull [11] tem-se que:

$$
\mathrm{F}(\leq \mathrm{m}): \mathrm{P}(\mathrm{f})=1-\exp \left[\left(\frac{\left(\sigma / \sigma_{\mathrm{m}}\right)^{w}}{\sigma_{0}}\right) v\right]
$$

onde, $\sigma_{m}$ é a tensão abaixo da qual não se constata ruptura, $\sigma_{0}$ é uma constante de adaptação escalar e o $w$ é o módulo de Weibull. Como comentado anteriormente, ainda podese ter a fração de fragmentos menores ou igual do que um determinado tamanho ou massa, $F(\leq m)$, para obtenção da probabilidade de falha $P(f)$, dada a partir da Equação E, por:

$$
\mathrm{F}(\leq \mathrm{m}): \mathrm{P}(\mathrm{f})=1-\exp \left[-\frac{\left(\mathrm{m} / \mathrm{m}_{0}\right)^{\gamma+1}}{\gamma+1}\right]
$$

em que o expoente fractal da fragmentação $\gamma$ também representa uma medida da tenacidade ao impacto do material. O histograma da probabilidade de fratura, $P(f)$, em função da massa dos fragmentos, dado na Equação G, é utilizado para se obter uma curva linear a partir da aplicação do logaritmo 
em ambos os lados dessa equação, nas variáveis $F(\leq m)$ e $p_{i}=m_{i} / m_{0}$, respectivamente, da seguinte forma:

$$
\ln [\ln (1-\mathrm{P}(\mathrm{f}))] \sim \log \left(\frac{\mathrm{m}}{\mathrm{m}_{0}}\right)
$$

ou seja,

$$
\ln [\ln (1-\mathrm{F}(\mathrm{s}))]=(\mathrm{y}+1) \ln \left(\mathrm{m} / \mathrm{m}_{0}\right)-\ln (\mathrm{y}+1)
$$

Como resultado da Equação I, é possível observar a tenacidade ao impacto de um material por meio do parâmetro $\gamma$, que mede a inclinação da curva linear obtida pelo logaritmo, e fazer a comparação entre diferentes materiais ensaiados. A inclinação desta reta, dada pelo coeficiente angular $\gamma+1$, indica que quanto menor for o valor de $\gamma$ mais tenaz ao impacto é o material, ou seja, ele possui uma maior capacidade de resistir ao impacto. Portanto, com a Equação I é possível fazer o levantamento dos dados da fragmentação por impacto em uma tabela conforme é mostrado na secção dos resultados. Desta forma, este trabalho teve como objetivo a caracterização fractal da superfície de fratura provocada por impacto mecânico em porcelanas triaxiais. Para isso, foi feita a avaliação da dimensão fractal de ruptura das porcelanas de três composições diferentes e também em comparação ao vidro e ao acrílico comerciais.

\section{MATERIAIS E MÉTODOS}

Este trabalho foi dividido em duas etapas para sua melhor estruturação: 1) produção e análise das porcelanas com diferentes composições; e 2) realização de ensaio de impacto mecânico, com aparelho construído em laboratório e com uma metodologia de baixo custo para a análise da probabilidade de fratura. Nessa segunda etapa, a análise de probabilidade de fratura foi feita também utilizando outros materiais (vidro e acrílico) para comparação dos resultados.

As matérias-primas utilizadas neste trabalho para obtenção das porcelanas foram: feldspato, quartzito Pianaro, caulim Hori, argila caulinítica e alumina A1000. A argila, o feldspato e o caulim foram inicialmente desaglomerados em moinho de bolas por $8 \mathrm{~h}$, numa razão de 2:1 em massa para meio de moagem:material. Apenas o quartzito foi moído por $24 \mathrm{~h}$. Posteriormente os pós moídos foram desaglomerados em almofariz e passados em peneira com abertura de 0,180 $\mathrm{mm}$, malha 80 (ABNT). As matérias-primas utilizadas foram caraterizadas quanto à sua composição química, por fluorescência de raios X (Shimadzu, EDX-700) e mineralógica, por difração de raios X em equipamento Shimadzu, XRD 6000, com condições da difração de $2 \theta$ variando de 5 a $90^{\circ}$, com velocidade de varredura de $2 \% \mathrm{~min}$, utilizando-se radiação $\mathrm{CuK} \alpha$. Os difratogramas de raios $\mathrm{X}$ foram identificados utilizando-se a base do programa Match Phase Identification from Powder Difraction File v. 1.11.

Para a preparação das composições das porcelanas estudadas, foi considerada a composição básica: $10 \%$ de argila; $25 \%$ de caulim; $35 \%$ de feldspato; e $30 \%$ de quartzi-
Tabela I - Composições estudadas (\% em massa). [Table I - Studied compositions (wt\%).]

\begin{tabular}{cccc}
\hline Composição & (1) Q30A0 & (2) Q15A15 & (3) Q0A30 \\
\hline Argila & 10 & 10 & 10 \\
Caulim & 25 & 25 & 25 \\
Feldspato & 35 & 35 & 35 \\
Quartzito & 30 & 15 & - \\
Alumina & - & 15 & 30 \\
\hline
\end{tabular}

to (composição 1: Q30A0). A partir dessa composição, o quartzito foi substituído por alumina, total e parcialmente, conforme se pode observar na Tabela I, a qual apresenta as formulações das composições estudadas. Na composição 2 (Q15A15), 50\% em massa do quartzito foi substituído pela alumina e na composição 3 (Q0A30) o quartzito foi totalmente substituído pela alumina. A razão para essa substituição foi modificar as propriedades mecânicas das porcelanas.

Os corpos de prova foram conformados por prensagem uniaxial em uma prensa hidráulica, num molde metálico com $60 \mathrm{~mm}$ de comprimento e $16 \mathrm{~mm}$ de largura, com pressão de $30 \mathrm{MPa}$. Depois de compactados, os corpos de prova foram sinterizados em forno elétrico (Jung), com taxa de aquecimento de $10^{\circ} \mathrm{C} / \mathrm{min}$ até 1150,1200 e $1250{ }^{\circ} \mathrm{C}$, com patamar de $2 \mathrm{~h}$. Os corpos de prova sinterizados foram caracterizados pelos ensaios de densidade aparente (DA), porosidade aparente (PA) e absorção de água (AA) usando-se a norma ASTM C373 [12] e medidas de resistência mecânica à flexão em três pontos, utilizando uma máquina universal de ensaios (Shimadzu, Autograph AGS, $10 \mathrm{kN}$ ). Para a verificação das fases formadas após sinterização, foi feita difração de raios $\mathrm{X}$, empregando-se o equipamento já mencionado. A microestrutura das porcelanas foi analisada por microscopia eletrônica de varredura (MEV), feita em microscópio da Tescan, Mira 3, após as amostras terem sido lixadas, polidas e atacadas quimicamente com solução de $5 \%$ de HF.

Após a análise das composições estudadas foi escolhida a melhor temperatura de sinterização para a produção dos corpos de prova para o ensaio de impacto mecânico. Para conformação destes corpos de prova, os pós foram depositados em molde metálico circular, com $50 \mathrm{~mm}$ de diâmetro, para compactação. Após isso o molde metálico foi levado a uma máquina universal de ensaios mecânicos (Shimadzu, AG-1, $300 \mathrm{kN}$ ), com a qual foram compactados sob pressão de aproximadamente $60 \mathrm{MPa}$. No total, foram feitos 15 corpos de prova para cada composição. Logo após, os corpos de prova foram sinterizados na temperatura determinada pelos ensaios anteriores.

As dimensões dos corpos de prova de vidro e de acrílico, que foram utilizados para a comparação dos resultados, dependeram dos resultados de dimensionamento da porcelana após a sua sinterização, devido à sua retração durante o processo de queima para, no final, se obter amostras com mesmo diâmetro. Assim, após sinterização, foram medidas as amostras de porcelanas, e o vidro e acrílico foram comprados com diâmetro de $45 \mathrm{~mm}$ e espessura de $3 \mathrm{~mm}$ de 
forma que as dimensões fossem iguais às das porcelanas.

O estudo do impacto mecânico consistiu de três partes principais: a construção de uma estrutura, na qual a ponta balística foi apoiada, a introdução de energia, ou seja, levantamento desta ponta para o impacto, e finalmente a sua queda e a consequente quebra do corpo de prova. $\mathrm{O}$ modelo de aparelho usado neste trabalho seguiu os modelos já existentes, descritos por outros autores, com características semelhantes, porém foram alterados alguns detalhes de acordo com as necessidades desse trabalho [11]. A Fig. 1 apresenta o equipamento construído para a realização do ensaio de impacto mecânico usado para se obter a probabilidade de fratura.

O ensaio consistiu em deslizar, por gravidade, a bala de impacto sobre os trilhos da barra de apoio a partir de uma dada altura $(h)$, até cair sobre o corpo de prova que ficou em repouso sobre o copo cilíndrico. Isso produziu a fragmentação do corpo de prova. A bala de impacto com massa $(m)$ de

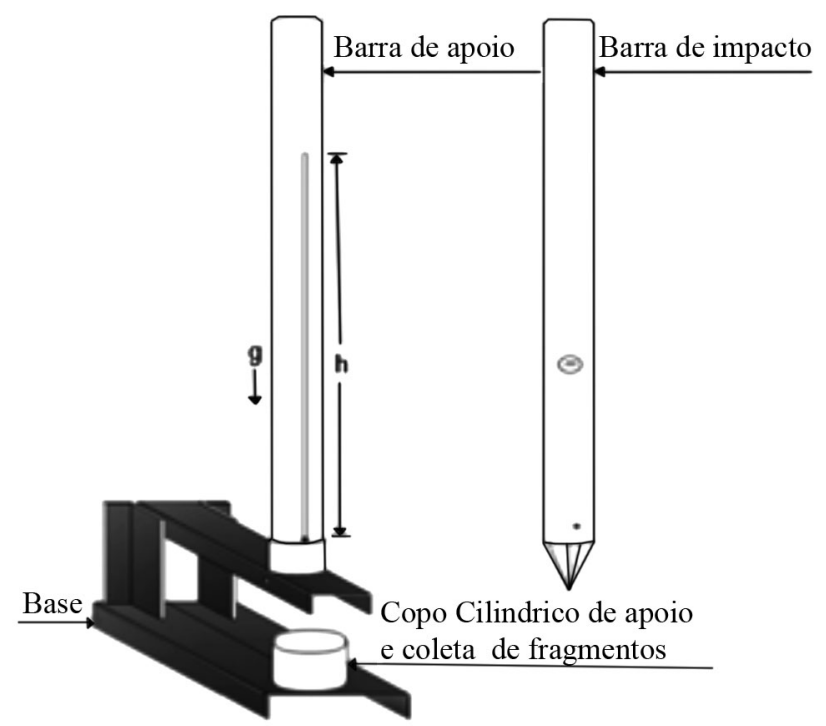

Figura 1: Esquema do aparelho de ensaio de impacto mecânico. [Figure 1: Schematic of mechanical impact test apparatus.]
2,125 $\mathrm{kg}$ continha uma ponta cônica. A altura h e a massa foram utilizadas no cálculo da energia do impacto imposta sobre o material. Para a realização do ensaio de impacto, foram feitas a pesagem e medidas dos corpos de provas, dos quais foram retirados os dados tais como, diâmetro, espessura e massa, e a massa da bala de impacto. Após isso, o equipamento foi preparado de forma adequada para que todos os procedimentos estivessem corretos, como: ajustes do equipamento, lubrificação da barra de apoio para diminuir o atrito entre a bala de impacto e a barra de apoio, proteção individual, e um anteparo ao redor do equipamento para que não houvesse perda de material quando feito o impacto. Os corpos de prova foram então colocados no equipamento e centralizados no copo cilíndrico. A bala de impacto foi levantada a uma dada altura, suficiente para a ruptura do material. A bala de impacto foi então solta para que ocorresse o choque da sua ponta com o corpo de prova, havendo assim a fratura do material. Posteriormente o material fragmentado foi recolhido e os pedaços fragmentados foram pesados e fotografados. Foram ensaiados 10 corpos de prova para cada material. A análise fractal da fratura por impacto das porcelanas, do vidro e do acrílico foi obtida por meio da análise estatística da fração de massa dos fragmentos e da probabilidade de falha dada pela fração de fragmentos menores do que um determinado tamanho, aqui representado pela massa $\mathrm{m}, F(\leq m)$, para uma mesma energia aplicada sobre o corpo de prova.

\section{RESULTADOS E DISCUSSÃO}

Na Tabela II está representada a composição química das matérias-primas, que foram caracterizadas por fluorescência de raios X. Pode-se observar que todas as matérias-primas, excetuando-se a alumina, apresentaram como um dos principais constituintes o $\mathrm{SiO}_{2}$. Como fundentes, pode-se observar a presença de óxido de sódio, de ferro, de potássio e de cálcio, com percentagens consideráveis. O quartzito foi a matéria-prima que possuiu o maior teor de sílica, com $96,94 \%$ e o feldspato apresentou teores altos de sílica e alumina na sua composição, e 6,34\% de óxido de sódio que auxiliou

Tabela II - Composição química (\% em massa) das matérias-primas em termos de óxidos.

[Table II - Chemical composition of the raw-materials in terms of oxides.]

\begin{tabular}{cccccc}
\hline Composição & Argila & Caulim & Feldspato & Quartzito & Alumina \\
\hline $\mathrm{SiO}_{2}$ & 48,51 & 45,41 & 65,22 & 96,94 & - \\
$\mathrm{Al}_{2} \mathrm{O}_{3}$ & 46,01 & 49,62 & 23,15 & 2,06 & 99,8 \\
$\mathrm{TiO}_{2}$ & 3,28 & 0,12 & 0,37 & 0,12 & - \\
$\mathrm{Fe}_{2} \mathrm{O}_{3}$ & 1,42 & 1,34 & 1,78 & - & 0,02 \\
$\mathrm{~K}_{2} \mathrm{O}$ & 0,61 & 2,32 & 1,82 & - & - \\
$\mathrm{CaO}$ & 0,15 & - & 1,30 & - & 0,02 \\
$\mathrm{Na} \mathrm{N}_{2}$ & - & - & 6,34 & - & 0,08 \\
$\mathrm{SO}_{3}$ & - & 1,11 & - & 0,35 & - \\
$\mathrm{ZrO}_{2}$ & - & - & - & 0,52 & - \\
$\mathrm{MnO}$ & - & 0,05 & - & - & - \\
\hline
\end{tabular}


no controle de vitrificação do corpo cerâmico. A alumina, que foi usada para substituição parcial e total do quartzo, apresentou a composição mais pura com $99,8 \%$ de $\mathrm{Al}_{2} \mathrm{O}_{3}$. O caulim, além da presença de $\mathrm{SiO}_{2}$ e $\mathrm{Al}_{2} \mathrm{O}_{3}$, apresentou óxido de potássio equivalente a $2,32 \%$, e óxidos de titânio e de ferro, equivalentes a $1,46 \%$.

As análises de difração de raios $\mathrm{X}$ mostraram que a argila apresentou as fases $\alpha$-quartzo, caulinita e ilita. O feldspato apresentou a fase albita e o caulim as fases $\alpha$-quartzo e também a caulinita. Na difração de raios $\mathrm{X}$ das matérias -primas que foram substituídas parcial ou totalmente, observou-se que a alumina apresentou somente picos referentes à $\alpha$-alumina e o quartzito apresentou somente a presença de picos relativos ao $\alpha$-quartzo. A Fig. 2 apresenta a variação da densidade aparente, porosidade aparente, absorção de água e resistência à flexão com a temperatura de sinterização para as três composições estudadas. Observou-se que as menores densidades e maiores porosidades aparentes e absorção de água foram encontradas na temperatura de $1150{ }^{\circ} \mathrm{C}$, na qual os corpos de prova ainda não sofreram mudanças significativas devido à sinterização. Com o aumento da temperatura de sinterização, a porosidade e a absorção de água diminuíram. Isso ocorreu devido à sequência de reações que ocorreram durante o processo de sinterização dos corpos de prova, como a formação de fase líquida proveniente do feldspato, a decomposição do caulim e a diminuição da viscosidade da fase líquida [13, 14]. As diferenças de densidades aparentes apresentadas entre as composições $1,2 \mathrm{e}$ 3 ocorreram devido ao aumento da quantidade de alumina nessas composições, uma vez que a alumina apresenta uma densidade teórica de $3,99 \mathrm{~g} / \mathrm{cm}^{3}$ enquanto que a do $\alpha$-quartzo é de $2,65 \mathrm{~g} / \mathrm{cm}^{3}$.

A Fig. 2 d mostra os resultados das medidas de resistência à flexão para as 3 composições em função da temperatura de sinterização. O maior valor encontrado foi na composição Q0A30 (30\% alumina), $139 \pm 13 \mathrm{MPa}$, decorrente da sinterização a $1250{ }^{\circ} \mathrm{C}$. Na composição A15Q15 (15\% alumina) o maior valor da resistência a flexão foi de $77 \pm 11 \mathrm{MPa}$ para o
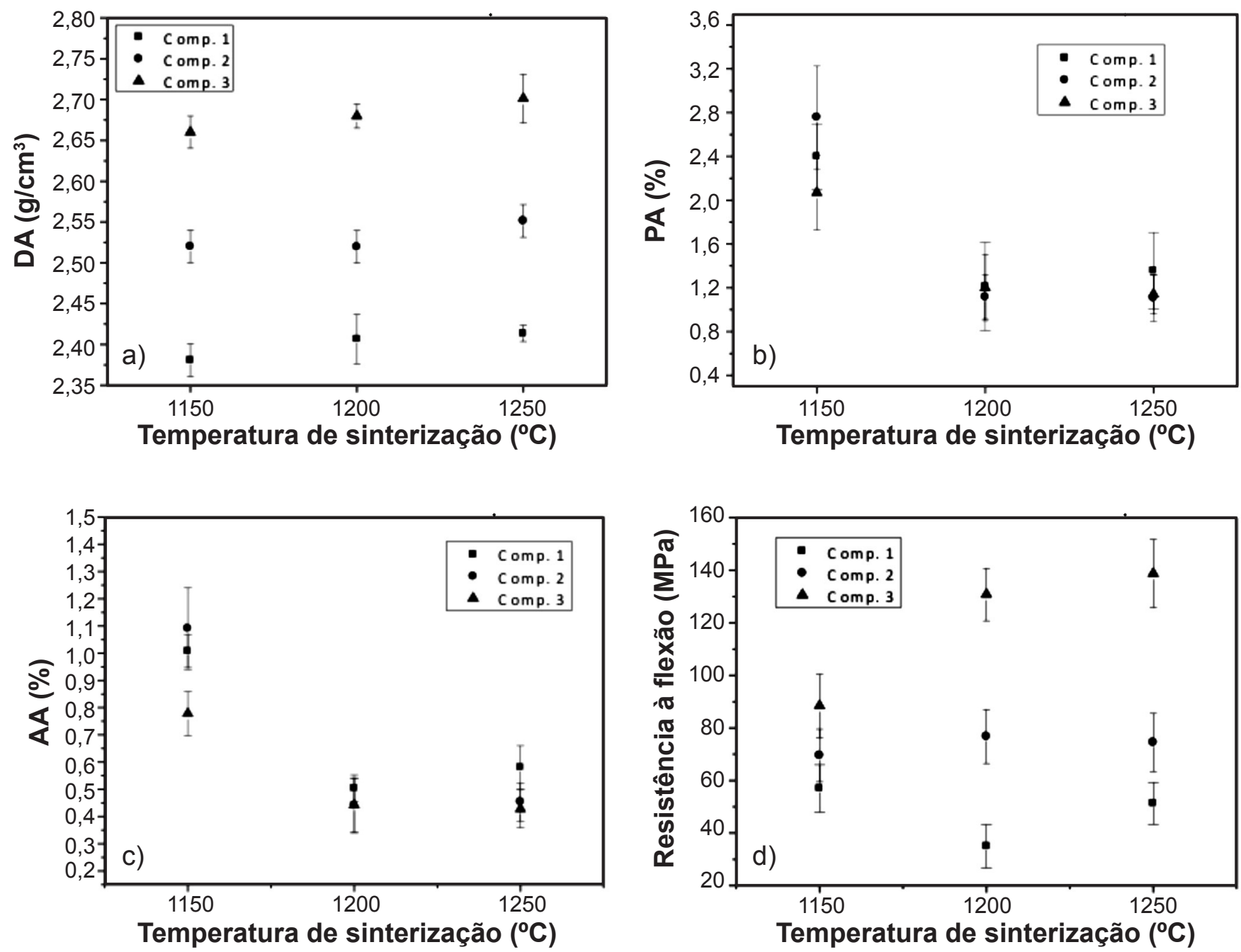

Figura 2: Densidade aparente (a), porosidade aparente (b), absorção de água (c) e módulo de ruptura à flexão (d) dos corpos de prova em função da temperatura de sinterização.

[Figure 2: Apparent density (a), apparent porosity (b), water absorption (c), and flexural strength (d) of samples as a function of sintering temperature.] 
material sinterizado a $1250^{\circ} \mathrm{C}$ e na composição A30Q0 $(30 \%$ quartzo) o valor encontrado foi de $57 \pm 9 \mathrm{MPa}$, também para a sinterização a $1250{ }^{\circ} \mathrm{C}$. Observou-se que as composições 2 e 3, que continham alumina, apresentaram valores de resistência mecânica maiores, sendo que a composição 3 , na qual o quartzo foi totalmente substituído pela alumina foi a que obteve os maiores valores de resistência mecânica em todas as temperaturas de sinterização.

Na Fig. 3 são apresentados os difratogramas de raios $\mathrm{X}$ das composições 1 , 2 e 3 sinterizadas a $1250{ }^{\circ} \mathrm{C}$, por estas apresentarem as maiores resistências mecânicas. Na composição 1 foram detectadas apenas as fases cristalinas da mulita e $\alpha$-quartzo. Nas composições 2 e 3 , foi possível observar, além dos picos de $\alpha$-quartzo e mulita, picos referentes à fase $\alpha$-alumina. Nas três composições foi possível também observar uma banda larga entre 15 e $30^{\circ}$, referente a uma fase amorfa, proveniente da fusão do feldspato. A presença de alumina provocou um aumento na

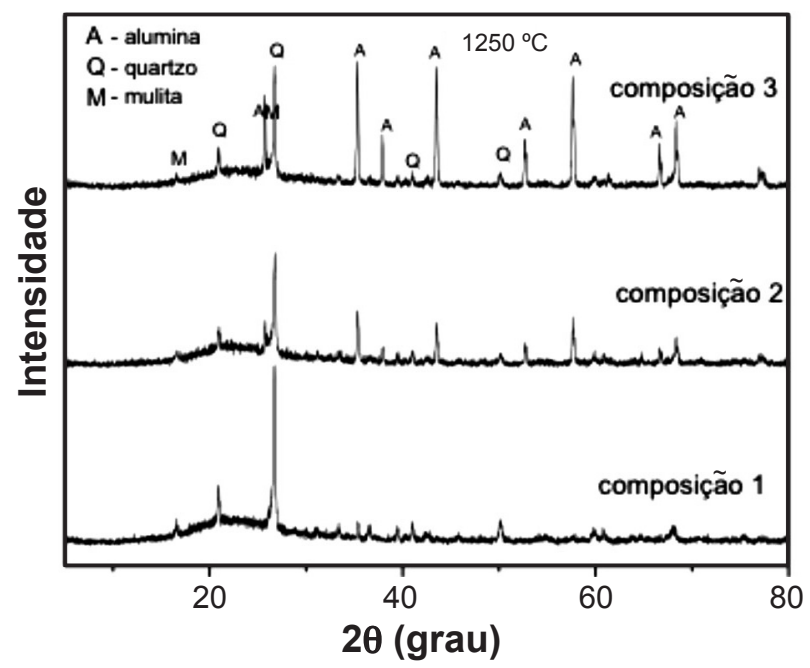

Figura 3: Difratogramas de raios $\mathrm{X}$ das composições 1, 2 e 3 sinterizadas a $1250^{\circ} \mathrm{C}$.

[Figure 3: X-ray diffraction patterns of compositions 1, 2 and 3 sintered at $1250^{\circ} \mathrm{C}$.]
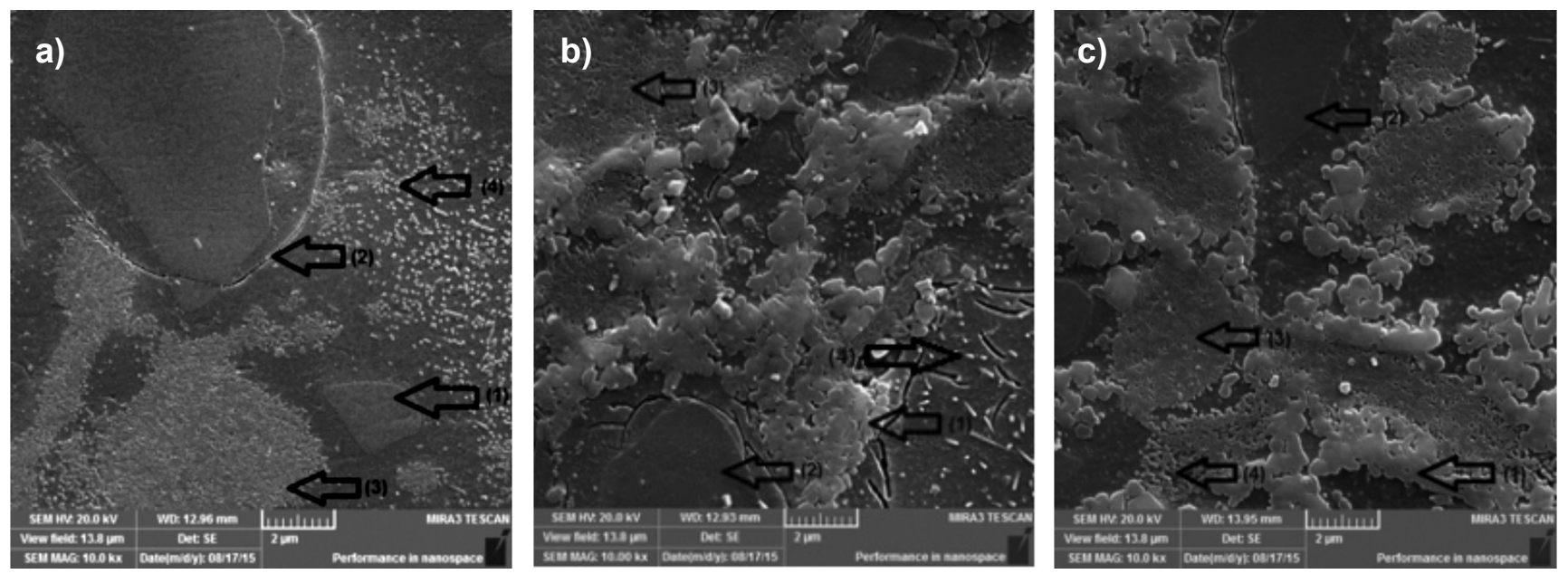

Figura 4: Micrografias obtidas por microscopia eletrônica de varredura dos materiais das composições 1 (a), 2 (b) e 3 (c) sinterizadas a $1250{ }^{\circ} \mathrm{C}$. [Figure 4: SEM micrographs of the compositions 1 (a), 2 (b), and 3 (c) sintered at $1250^{\circ} \mathrm{C}$.] resistência mecânica, provavelmente pelo mecanismo de reforço por dispersão de partículas e por possuir um maior módulo elástico que o quartzo, uma vez que o módulo elástico da alumina é $380 \mathrm{GPa}$, enquanto que o do quartzo é $70 \mathrm{MPa}$. As partículas dispersas na matriz vítrea atuaram no sentido de barrar a propagação das trincas. Além disso, a substituição do quartzo, que possui coeficiente de expansão térmica de $23 \times 10^{-6} \mathrm{~K}^{-1}$ no intervalo de temperatura entre 20 e $700{ }^{\circ} \mathrm{C}$, por alumina, que possui coeficiente de expansão térmica de $8 \times 10^{-6} \mathrm{~K}^{-1}$, no mesmo intervalo de temperatura, reduziu a diferença do coeficiente de expansão térmica entre a fase vítrea $\left(3 \times 10^{-6} \mathrm{~K}^{-1}\right)$ e a fase cristalina; dessa forma, a possibilidade de trincamento decresceu e a resistência mecânica da porcelana foi aumentada $[15,16]$.

A microestrutura das composições 1,2 e 3 sinterizadas a $1250{ }^{\circ} \mathrm{C}$ estão apresentadas na Fig. 4. Pode-se observar nas três micrografias a presença da fase vítrea na qual estão dispersas partículas de quartzo e/ou de alumina e regiões ricas em mulita primária e mulita secundária [4]. Na Fig. 4a observa-se a micrografia da composição 1 , que mostra que as partículas de quartzo possuem bordas arredondadas devido à sua dissolução na fase vítrea (flecha 1). Nota-se também o trincamento em volta das partículas de quartzo, decorrentes das diferenças de coeficientes de expansão térmica da matriz e das partículas de quartzo (flecha 2, Fig. 4a) [14]. A mulita primária se apresentou na forma de cristais agregados de pequenas dimensões, e foram provenientes da transformação da caulinita, presente nas matérias-primas (flecha 3, Fig. 4a). A mulita secundária se apresentou na forma de agulhas prismáticas, formada na região do feldspato (flecha 4, Fig. 4a). As microestruturas das composições $2 \mathrm{e}$ 3, mostradas nas Figs. 4b e 4c, apresentaram partículas aglomeradas de alumina (flecha 1), partículas de quartzo (flecha 2), mulita primária (flecha 3) e mulita secundária (flecha 4) dispersas numa fase vítrea. Pôde-se observar também que, mesmo com a substituição total do quartzito pela alumina na composição 3, houve a presença de partículas de quartzo na microestrutura. Essas partículas de quartzo foram provenientes das outras matérias-primas utilizadas na produção 
dessas porcelanas. A presença de mulita secundária nas micrografias das composições 2 e 3 foi bem menor que na composição 1 . De acordo com $[14,15]$, durante a sinterização, a mulita secundária é formada a partir da argila caulinítica e cresce em direção ao líquido originado pelo feldspato. Assim, o fato das partículas de alumina terem se aglomerado, provavelmente devido ao seu pequeno tamanho de partícula inicial, e se posicionado, durante a sinterização, ao redor das partículas de mulita primária, pode ter impedido o crescimento da mulita secundária. A aglomeração das partículas de alumina ao redor da mulita primária pode ser observada na Fig. 4c.

Após a caracterização dos corpos de prova das três composições das porcelanas, observou-se pelos resultados obtidos que as melhores propriedades para as três composições foram atingidas na temperatura de sinterização de $1250{ }^{\circ} \mathrm{C}$. Dessa forma, a análise fractal foi feita nas porcelanas sinterizadas a $1250{ }^{\circ} \mathrm{C}$, para as quais apresentam-se as maiores diferenças. Os resultados obtidos no estudo do impacto estão apresentados na Fig. 5, na qual foram registradas a soma quantitativa e a média de todos os resultados obtidos, para cada uma das três composições das porcelanas sinterizadas a $1250{ }^{\circ} \mathrm{C}$ e para os materiais de comparação (vidro e acrílico). Os valores para as amostras testadas mostraram a tendência de resistência à fratura, dado pela fração de fragmentos $F(\leq m)$ mais leves do que uma dada massa $m$, em função da massa relativa dos fragmentos $\left(\mathrm{m} / \mathrm{m}_{0}\right)$, dada pela Equação G. Estes resultados foram calculados através da probabilidade de falha $\left(\mathrm{P}_{\mathrm{f}}\right)$, na qual se observou, a partir dos valores obtidos, que as porcelanas se encontraram entre os resultados do vidro e do acrílico, apresentando semelhança quanto ao número de fragmentos. Dentre as três composições de porcelanas estudadas, a composição 3 foi a que mostrou maior desempenho, estando mais próxima ao acrílico. Isto se deu devido à rigidez da estrutura da porcelana já discutido anteriormente.

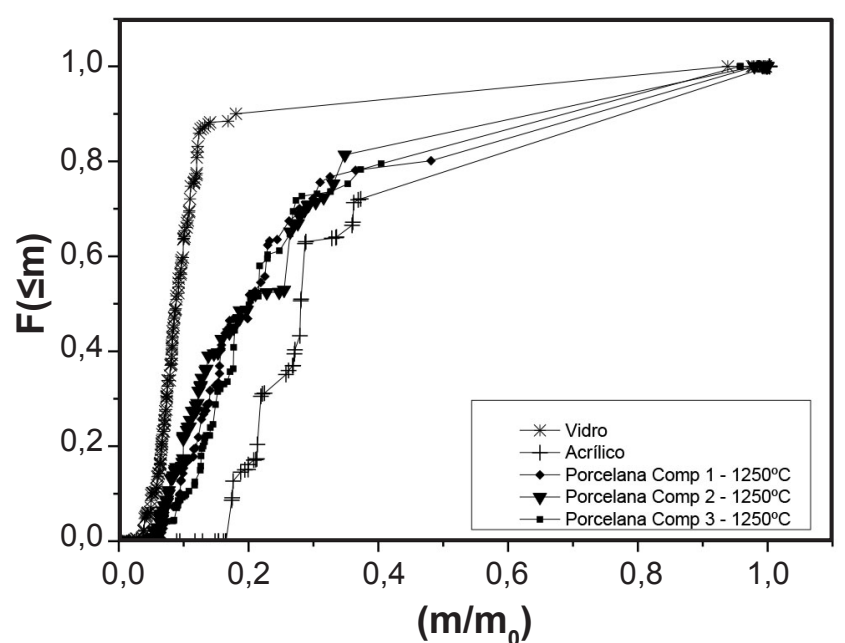

Figura 5: Frequência $F(\leq m)$ do tamanho dos fragmentos em função da massa relativa $\left(\mathrm{m} / \mathrm{m}_{0}\right)$.

[Figure 5: Frequency $F(\leq m)$ of fragment size depending upon the relative mass $\left.\left(\mathrm{m} / \mathrm{m}_{0}\right) \cdot\right]$
Segundo [17], os fragmentos das porcelanas têm as mesmas características geométricas e dimensões fractais, os quais são determinados pelo método das ilhas cortadas (slit island method). Sendo assim, observou-se a semelhança do número de fragmentos e nas características das curvas das porcelanas para diferentes composições após o impacto.

A fragilidade das porcelanas depende da microestrutura que elas apresentam. As trincas e poros presentes na microestrutura podem afetar diretamente o comportamento da resistência ao impacto, sendo que a fratura começa com a sua dissipação através destas falhas. A análise fractográfica por MEV revelou a real geometria da trinca e da fragmentação. A metodologia usada na análise fractal por impacto mecânico trouxe como resultado a revelação de parâmetros diferentes da medida de resistência à flexão em três pontos [18]. Estas duas análises são obviamente diferentes, contudo, foi possível por meio da análise fractal da fragmentação entender um pouco mais dos mecanismos envolvidos no processo de fragmentação, o que não foi possível com um simples ensaio de flexão. Pôde-se verificar que o vidro teve tendência a ter um número elevado de fragmentos pequenos, isto pela fragilidade do material e a energia que este dissipa. Isto está de acordo com o fato do vidro ter uma baixa energia total de fratura, cerca de 3,5 a $5,3 \mathrm{~J} / \mathrm{m}^{2}$, o que se traduz em grande superfície de fratura, para um dado valor de energia elástica armazenada durante o ensaio de impacto. Isso, por sua vez, significa que uma grande quantidade de fragmentos é produzida para gastar a energia total de fratura transmitida pelo impacto. Em comparação aos outros materiais, o número de fragmentos foi, portanto, maior. Observou-se que isto foi diretamente relacionado à estrutura amorfa do vidro. No caso do acrílico, este parâmetro de maior resistência e menor dissipação foi ligado ao tamanho dos esferulitos [19]. Em todas as amostras observaram-se trincas radiais, que partiram do centro da amostra e se dirigiram até a borda. $\mathrm{O}$ acrílico foi o material que absorveu mais o impacto, sendo que a energia foi distribuída a partir da superfície que recebeu o impacto para a superfície oposta, ou seja, a energia se dissipou de cima para baixo da amostra, e posteriormente foi transmitida para as bordas. Ele foi o material que menos fragmentou, devido à sua maior tenacidade ao impacto e maior capacidade de reter a energia. Observou-se também que o padrão de trincas formado pelo acrílico, o número de ramos da trinca e consequentemente o número de fragmentos, seguiu um padrão de acordo com os modos normais de vibração de uma membrana circular.

Para melhor observar os parâmetros de análise fractal da fragmentação dos materiais, foi necessário o estudo dos expoentes fractais da fragmentação das curvas da Fig. 5. Esses expoentes relacionados à tenacidade ao impacto foram calculados por meio de uma escala logarítmica a partir dos valores apresentados anteriormente na Fig. 5. Nesta representação foi usada a Equação I e está apresentada na Fig. 6, que mostra a comparação dos resultados obtidos das três composições de porcelanas sinterizadas a $1250^{\circ} \mathrm{C}$, do vidro e do acrílico. Para uma melhor observação foi usada uma mesma escala. Um ajuste linear para uma reta do tipo 

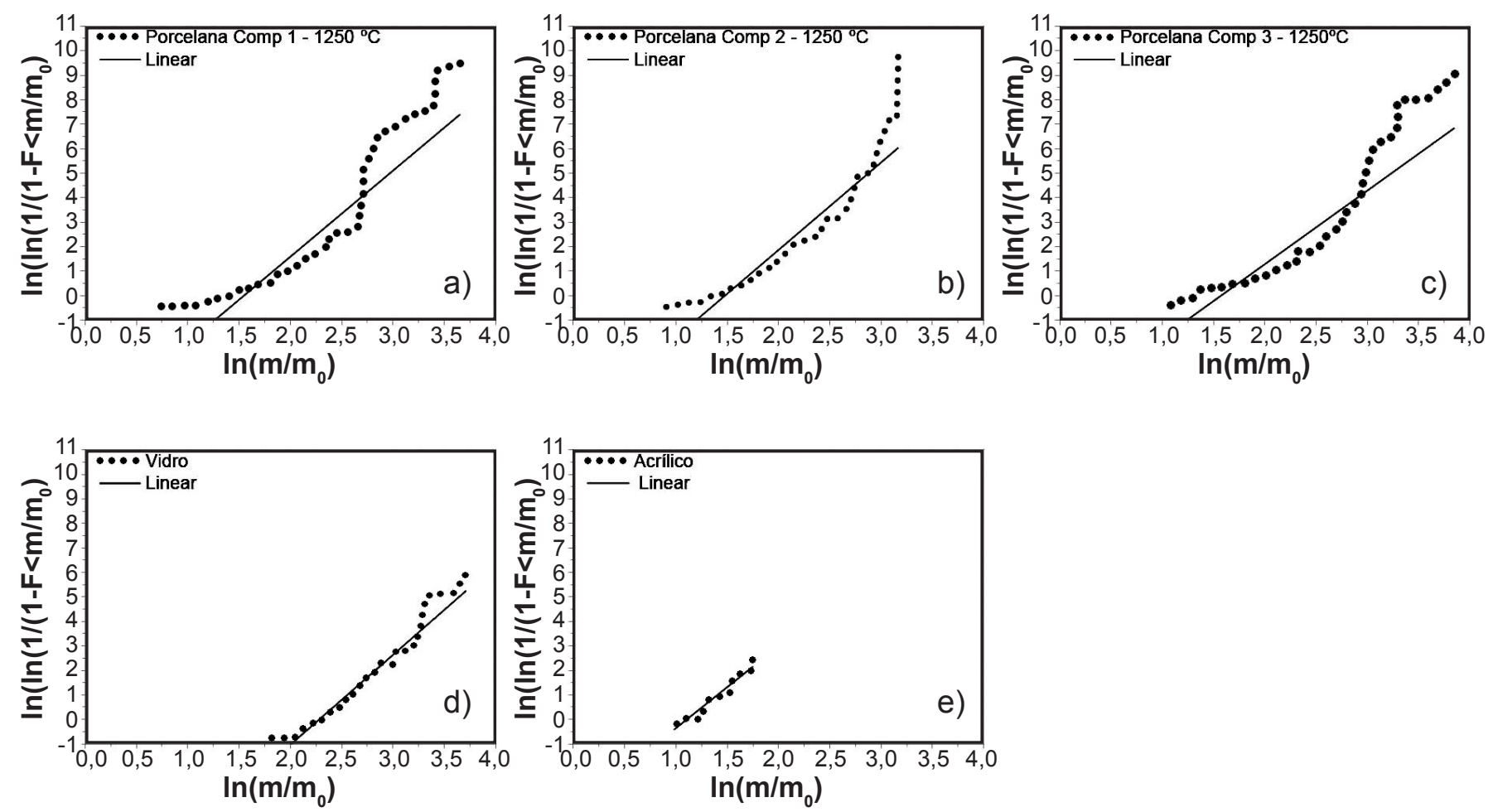

Figura 6: Comparação da tendência da tenacidade ao impacto entre os materiais estudados.

[Figure 6: Comparing the trend of impact toughness of the studied materials.]

Tabela III - Análise fractal da fratura por impacto central.

[Table III - Fractal analysis of fracture by central impact.]

\begin{tabular}{lcccc}
\hline Material & $\begin{array}{c}\text { Coeficiente angular } \\
(\mathrm{a}=\gamma+1)\end{array}$ & $\begin{array}{c}\text { Coeficiente linear } \\
{[\mathrm{b}=-\ln (\gamma+1)]}\end{array}$ & $\begin{array}{c}\text { Coeficiente de } \\
\text { correlação }(\mathrm{R})\end{array}$ & $\begin{array}{c}\text { Número de } \\
\text { fragmentos }\end{array}$ \\
\hline Vidro & $3,67 \pm 0,06$ & $-8,38 \pm 0,17$ & 0,9651 & $11-15$ \\
Porcelana 1 (Q30A0) & $3,51 \pm 0,19$ & $-5,44 \pm 0,42$ & 0,8315 & $6-8$ \\
Porcelana 2 (Q15A15) & $3,57 \pm 0,18$ & $-5,28 \pm 0,37$ & 0,8666 & $6-8$ \\
Porcelana 3 (Q0A30) & $3,01 \pm 0,16$ & $-4,73 \pm 0,37$ & 0,8306 & $6-9$ \\
Acrílico & $3,34 \pm 0,15$ & $-3,71 \pm 0,20$ & 0,9444 & $4-5$ \\
\hline
\end{tabular}

$\mathrm{y}=\mathrm{ax}+\mathrm{b}$ foi realizado utilizando o método dos mínimos quadrados. Através destes dados, foi possível calcular os coeficientes angulares, $a=\gamma+1$, o coeficiente linear $\mathrm{b}=\ln (\gamma+1)$ e o coeficiente de correlação. Este ajuste linear é uma primeira aproximação da análise estatística sugerida pela teoria fractal da fragmentação [10]. Para o vidro e o acrílico este ajuste linear pareceu razoavelmente adequado fornecendo os dados necessários para a análise comparativa dos resultados com outros materiais. Contudo, observou-se que para as amostras de porcelana a correlação linear não foi a mais adequada. Por esta razão sugere-se a aplicação de uma estatística não linear em um artigo futuro para melhor expressar os resultados obtidos para as porcelanas. A contagem aproximada do número dos fragmentos das amostras foi realizada para se ter uma ideia do grau de entropia do fenômeno da fragmentação e para comparar entre si com os números de fragmentos dos outros materais.
Os valores calculados estão listados na Tabela III.

A Tabela III apresenta os resultados da análise fractal do impacto de três formas diferentes: o coeficiente angular diz respeito ao grau de resistência ao impacto, o coeficiente linear diz respeito ao grau de confiabilidade da peça e o número de fragmentos diz respeito ao grau de distribuição de energia, conforme explicado na sequência. Se a reta da análise de impacto possui um coeficiente angular mais inclinado, consequentemente o material possui uma tenacidade ao impacto menor e, portanto, é mais frágil. O coeficiente linear indica a confiabilidade da peça, pois quanto menor o coeficiente linear "mais confiável" é a tensão de fratura e, portanto, mais próximo do valor desejado a peça tem em todas as suas partes. O número de fragmentos indica a distribuição de energia total de fratura no material. Sendo o vidro o material que se fragmentou mais, ele teve a capacidade de transformar mais a energia do impacto em fragmentos, ao 
contrário do acrílico que reteve mais a energia do impacto transformando em outras formas de dissipação do que em fragmentos. Por se tratar de porcelanas de diferentes composições, os valores apresentados mostraram a diferença entre elas. Observou-se que a composição 3 da porcelana, na qual todo o quartzito foi substituído pela alumina, foi a que apresentou uma menor inclinação, indicando assim que ela possuiu uma maior tenacidade ao impacto que as outras composições. Essa composição foi a que também apresentou a maior resistência mecânica à flexão.

Os valores da Tabela III também mostraram que, de todos os materiais analisados, o vidro teve um coeficiente angular maior do que os demais. Isto está de acordo com a sua tenacidade ao impacto inferior e indicou que o vidro, sendo mais frágil, possui também uma maior distribuição de energia, pois se fragmentou em um maior número de fragmentos sendo menos resistente à fratura. As porcelanas em relação ao vidro foram mais resistentes ao impacto, como mostram os coeficientes angulares ligeiramente menores e, portanto, um pouco mais tenazes com tensão de fratura também maior. Elas também se fragmentaram com um número intermediário de fragmentos em relação ao vidro e ao acrílico distribuindo, portanto, a energia de impacto de forma intermediária. Já o acrílico apresentou uma maior tenacidade ao impacto do que as porcelanas 1 e 2, e uma menor tenacidade do que a porcelana 3. Ele também se fragmentou em um menor número de fragmentos distribuindo menos a energia na forma de fragmentos por causa da sua alta energia total de fratura que é da ordem de 140-400 J/ $\mathrm{m}^{2}$. O coeficiente angular e também o coeficiente linear da Equação I estão

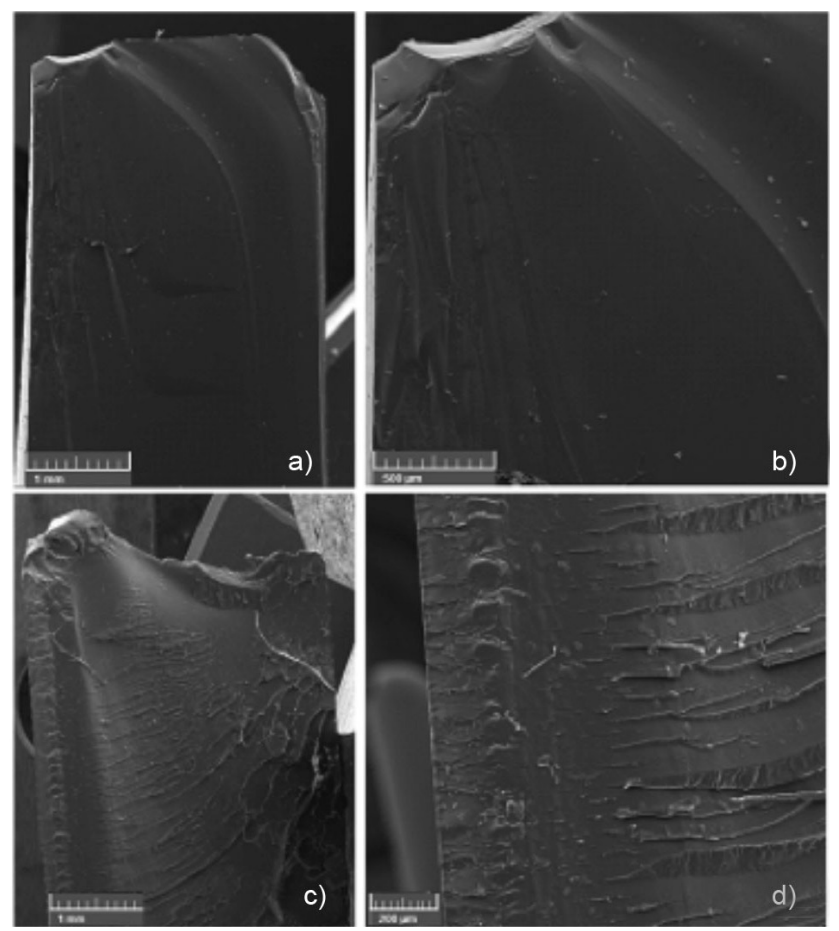

Figura 7: Micrografias das superfícies fraturadas do vidro $(a, b)$ e acrílico (c, d).

[Figure 7: Fracture surface micrographs of glass (a, b) and acrylic $(c, d)$.] ambos relacionados ao valor $(\gamma+1)$ e ao expoente de Weibull da Equação $\mathrm{F}$, porém de uma forma indireta, visto que as relações entre estas duas equações não são diretas. Como a análise é feita para uma única peça que se fragmenta, a interpretação da análise associada à estatística de Weibull, neste caso, deve ser feita em relação às condições internas de uma única peça. Ela está relacionada à confiabilidade da tensão de fratura dentro do material e em relação ao número de fragmentos que se pode obter pelo impacto. Ou seja, na análise fractal da fragmentação, ao contrário de Weibull, quanto menor o coeficiente linear "mais confiável" é a tensão de fratura e, portanto, mais próximo do valor desejado a peça tem em todas as suas partes. Os valores do coeficiente de correlação mostram quão diretamente o gráfico se aproxima de uma reta. No caso os comportamentos do vidro e do acrílico foram mais próximos de uma reta do que os das porcelanas. O número de fragmentos está relacionado à entropia e diz se o material distribui mais ou menos a energia do impacto. Neste caso o vidro se fragmentou mais do que a porcelana e o acrílico e, portanto, distribuiu mais a energia do impacto com um maior número de pequenos fragmentos. Por meio dos resultados comparativos, a metodologia utilizada se mostrou viável para a caracterização do padrão fractal de uma fratura ramificada, para a caracterização da resistência mecânica ao impacto e para a caracterização dos parâmetros de fragmentação por impacto balístico.

Na Fig. 7 são apresentadas as micrografias das superfícies de fratura do vidro e do acrílico. Nas Figs. 7a e 7b observam-se a superfície de fratura do vidro, a qual apresentou as características de fratura padronizadas e já descritas por alguns autores. Segundo [20], as superfícies de fratura do vidro se distinguem por três áreas características: área de espelho, área cinzenta e área fibrosa. Nas imagens das Figs. $7 \mathrm{c}$ e $7 \mathrm{~d}$ observam-se micrografias da superfície fraturada do acrílico. O comportamento do acrílico foi diferente dos outros dois materiais, já que a energia aplicada sobre o acrílico foi dispersada do centro para as bordas, o que gerou a tendência de ranhuras arredondadas formadas pelos esferulitos, de dentro para fora [19]. Na parte oposta ao impacto, pôdese observar uma camada menor de formação de ranhuras, isto devido à pouca energia de impacto no fundo da amostra.

Na Fig. 8 estão apresentadas as micrografias da superfície de fratura da porcelana (composição 3), onde estão ampliadas sequencialmente. Pode-se observar que a superfície de fratura da porcelana mostra rugosidade e que o trajeto da trinca seguiu os poros existentes, fazendo camadas conforme mostra a Fig. 8b. A partir das micrografias das Figs. 7 e 8 pode-se observar a textura das superfícies de fratura e concluir que seu aspecto se reflete no valor do expoente de fragmentação $\gamma$. O vidro, por ser um material com menor energia total de fratura, transformou a energia do impacto diretamente em fragmentos, com um valor de $\gamma$ maior e uma superfície de fratura praticamente lisa. Já o acrílico, por ter um valor de energia total de fratura maior do que a do vidro, transformou a energia de impacto em um número menor de fragmentos, com um valor de $\gamma$ menor do que o vidro e a porcelana (Tabela III), apresentando uma superfície de fratura 

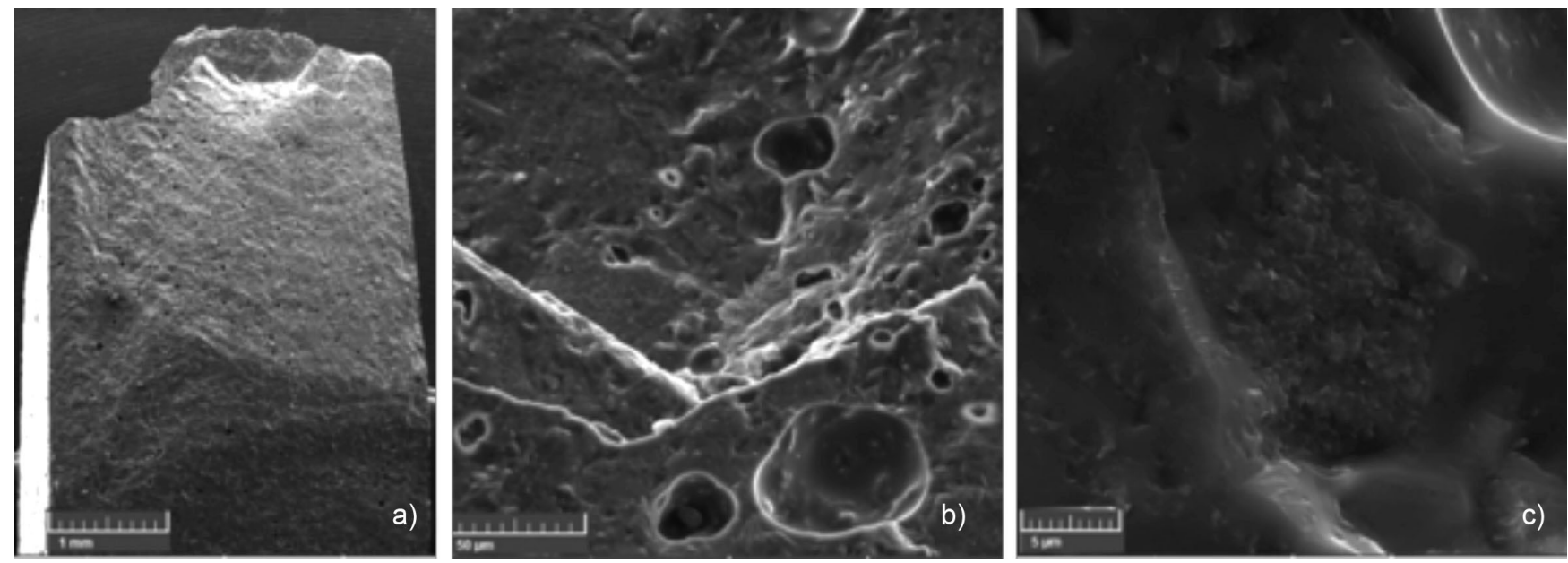

Figura 8: Micrografias das superfícies fraturadas da porcelana de composição 3 sinterizada a $1250{ }^{\circ} \mathrm{C}$.

[Figure 8: Fracture surface micrographs of the porcelain, composition 3, sintered at $1250^{\circ} \mathrm{C}$.]

mais rugosa. A porcelana por ter um valor de energia total de fratura intermediário produziu um número de fragmentos entre o vidro e o acrílico, com um valor de $\gamma$ também intermediário (Tabela III), com uma rugosidade mediana das superfícies de fratura entre o vidro e o acrílico.

\section{CONCLUSÕES}

Com base nos resultados obtidos nos estudos de impacto mecânico, conclui-se que: i) a substituição do quartzito pela alumina na composição da porcelana, tanto parcial quanto totalmente, acarretou em aumento de resistência mecânica, sendo que o maior valor foi de $139 \mathrm{MPa}$, alcançado pela composição 3 , na qual o quartzito foi substituído totalmente pela alumina; ii) verificou-se que a porcelana nas composições estudadas, possuiu uma tenacidade ao impacto que se situou entre a do vidro e a do acrílico; isto foi representado pelo expoente de fragmentação da porcelana que apresentou valor 3 a $20 \%$ menor que o do vidro e 7 a $10 \%$ maior do que o do acrílico; iii) o número de ramificações das trincas dependeu do tipo de material e foi relacionado com a distribuição da energia do impacto, o que resultou em um maior ou menor número de fragmentos; iv) observou-se que o número e a forma das trincas seguiram um padrão ramificado que dependeu do tipo de material; v) a metodologia se mostrou viável para a caracterização do padrão fractal de fratura ramificada, para a caracterização da resistência mecânica ao impacto e para a caracterização dos parâmetros de fragmentação por impacto balístico. Pôde-se concluir que o expoente de fragmentação por impacto $\gamma$ de um material é o fator determinante na resistência ao impacto.

\section{REFERÊNCIAS}

[1] Y.M Chiang, D. Birnie III, W.D. Kingery, Physical ceramics, John Wiley\& Sons, New York (1997) 343.
[2] J.S. Liebermann, Am. Ceram. Soc. Bul. 81, 2 (2002) 33.

[3] J.S. Liebermann, Am. Ceram. Soc. Bul. 80, 7 (2001) 43.

[4] A.L. Chinelatto, D.P.F. Souza, Cerâmica 50, 313 (2004) 62.

[5] A.L. Chinelatto, D.P.F. Souza, Cerâmica 50, 315 (2004) 172.

[6] R.G. Orlova, V.D. Beshentsev, J.K. Moroz,E.P. Bogdanis, Glass Ceram. 45, 11/12 (1988) 466-469.

[7] J. Liebermann, Am. Ceram. Soc. Bull. 80, 6 (2001) 37.

[8] R.C. Falcão, F. Parisio, App. Phy. Let. 105 (2014) 124102.

[9] W. Brown, J. Astrophy, Astron. 10 (1989) 89.

[10] W.K. Brown, K.H. Wohletz, J. Appl. Phys. 78 (1995) 2758.

[11] R. Eisenstadt, C. McCullough, in: ICF6, New Delhi (India) 1984 (2013).

[12] ASTM C373 "Standard test method for water absorption, bulk density, apparent porosity, and apparent specific gravity of fired whiteware products, ceramic tiles, and glass tiles" (2014).

[13] S.R. Bragança, C.P. Bergmann, Cerâmica 50, 314 (2004) 145.

[14] J. Martín-Márquez, J.M. Rincón, M. Romero, Ceram. Int. 34 (2008) 1867.

[15] Y. Iqbal, W.E. Lee, J. Am. Ceram. Soc. 82, 12 (1999) 3584.

[16] Y. Iqbal, W.E. Lee, J. Am. Ceram. Soc. 83, 12 (2000) 3121.

[17] Y. Zhiyuan, Z. Anning, J. Wuhan Univer. Tech. 20 (2005) 37.

[18] A. Celli, A. Tucci, L. Esposito, C. Palmonari, J. Eur. Ceram. Soc. 23 (2003) 469.

[19] L. Akcelrud, Fundamentos da ciência dos polímeros, Ed. Manole (2007).

[20] M.H. Rivera, L.C. Guerrero, in: Ingenierías IV (2001) 27.

(Rec. 29/01/2016, Rev. 24/06/2016, Ac. 26/09/2016) 